\title{
F-transform and discrete convolution
}

\author{
Pavel Vlašánek ${ }^{1}$ Irina Perfilieva ${ }^{2}$
}

\author{
${ }^{1,2}$ University of Ostrava, Institute for Research and Applications of Fuzzy Modeling, Ostrava, Czech Republic
}

\begin{abstract}
Discrete convolution is commonly used operation in the image processing field. The technique modifies an input image in various ways by scalable and easy computation. We are able to achieve many applications such as edge detection, noise reduction or artistic filtering, by selection of the proper kernel. The technique of the F-transform investigated in few last years also modifies an input image in the certain ways. Following paper introduces image processing based on the convolution operation and F-transform approximation with emphasis on similarities and negotiability of the both techniques. As an illustration, we will use the Gaussian convolution kernel and appropriate F-transform basic function for simple noise reduction.
\end{abstract}

Keywords: F-transform, convolution, image processing

\section{Introduction}

We propose the technique of F-transform as a universal technique for image processing. For this purpose, we draw a parallel with another universal technique, which is based on the convolution operation. The latter is commonly used together with specified kernels, which are chosen depending on a main processing task: noise removal [1], edge detection [2, 3], etc.

On the other hand, the F-transform is introduced as a technique, which maps a continuous/ discrete function onto a finite vector of its F-transform components. It has been shown that F-transform can be applied in image processing for edge detection [4], image filtering [5], image fusion [6], noise reduction [7], etc.

The purpose of this contribution is to show a universality of the F-transform by the demonstration of it relationship with the convolution. In details, we aim to show that the main parameter of the Ftransform technique (fuzzy partition) can be characterized with the help of a set of translations of a certain kernel function. This puts a bridge between both techniques.

The preliminary step in this direction was made in [7] where a comparison with the F-transform and Fourier transform was analyzed. In this contribution, we want to raise importance of a proper choice of a kernel with the correspondence to a task of image processing. This correspondence was in a shadow due to the fact that the F-transform works as a universal approximating tool independently of a shape of its partition elements (called basic functions) [8].

In particular, we will be focused on the task of noise reduction, where both techniques have confirmative results. Other applications of convolution and the F-transform will be analyzed in the foregoing publications.

The paper has the following structure: section 2 characterizes a noise reduction; section 3 describes basics of the F-transform and section 4 describes basics of the convolution. Their relationship is studied in section 5 . Conclusion is in section 6 .

\section{Noise reduction}

The noise reduction is comprehensive topic beyond the scope of this paper. However, let us describe some general features. A noise is typically viewed as the unwanted high frequencies included in the input function. Our goal, eliminate a noise, can be identified with these high frequencies elimination. For that purpose, a low-pass filter can be applied. It means that only low frequencies are passed to the output function. It has been proven that Ftransform works as low-pass filter [7] thus can be used for the noise reduction.

The high frequencies elimination can be achieved also via blurring. The example for case when the input function is identified with the image is in Fig. 1 and Fig. 2. The convolution operation using Gaussian kernel blurs input function thus can be used for the noise reduction also.

In the latter text, comparison is presented hand to hand with the RMSE result estimation.

\section{Discrete F-transform}

Below, we recall the definition of a fuzzy partition. Fuzzy sets $A_{0}, \ldots, A_{m} ; m<M$ identified with their membership functions (basic functions) $A_{0}, \ldots, A_{m}:[0, M] \rightarrow[0,1]$, establish a fuzzy partition of $[0, M]$ with nodes $0=x_{0}<x_{1}<\cdots<x_{m}=$ $M$ if the following conditions are fulfilled:

1) $A_{k}:[0, M] \rightarrow[0,1], A_{k}\left(x_{k}\right)=1$;

2) $A_{k}(x)=0$ if $x \notin\left(x_{k-1}, x_{k+1}\right), k=0, \ldots, m$;

3) $A_{k}(x)$ is continuous;

4) $A_{k}(x)$ strictly increase on $\left[x_{k-1}, x_{k}\right]$, $k=1, \ldots, m ;$ and strictly decrease on $\left[x_{k}, x_{k+1}\right], k=1, \ldots, m$

5) $\sum_{k=0}^{m} A_{k}(x)=1, x \in[0, M]$. 


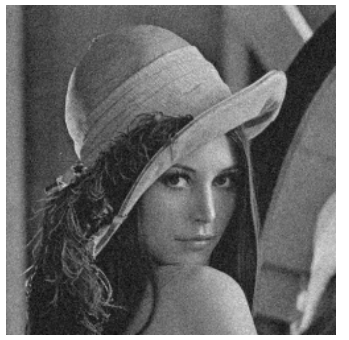

(a) Original

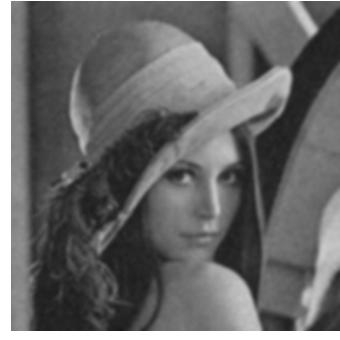

(b) Convolution

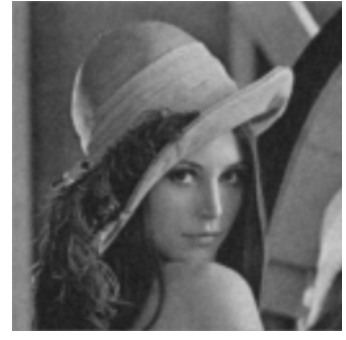

(c) F-transform
Figure 1: Original noisy image processed by a convolution with usage of the Gaussian filter and Ftransform.

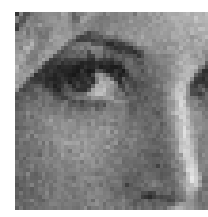

(a) Original

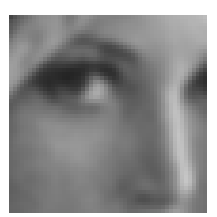

(b) Convolution

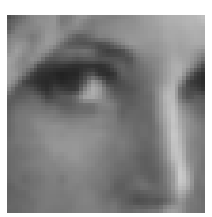

(c) F-transform
Figure 2: Detail of the Fig. 1.

We say that the fuzzy partition given by $A_{0}, \ldots, A_{m}$, is an $h$-uniform fuzzy partition if nodes $x_{k}=h k, k=0, \ldots, m$, are equidistant, $h=M / m$ and two additional properties are met:

6) $A_{k}\left(x_{k}-x\right)=A_{k}\left(x_{k}+x\right), x \in[0, h], k=$ $0, \ldots, m$

7) $A_{k}(x)=A_{k-1}(x-h), \quad k=1, \ldots, m, x \in$ $\left[x_{k-1}, x_{k+1}\right]$.

Parameter $h$ will be referred to as a radius. Assume that fuzzy sets $A_{0}, \ldots, A_{m}$ establish a fuzzy partition of $[0, M]$ and $I: P \rightarrow \mathbb{R}$ is a discrete real valued function defined on the set $P=\{x \mid x=$ $0,1, \ldots, M\}$. The following vector of real numbers $\mathbf{F}_{m}[I]=\left(F_{0}, \ldots, F_{m}\right)$ is the (direct) discrete $F$ transform of $I$ w.r.t. $A_{0}, \ldots, A_{m}$ where the $k$-th component $F_{k}$ is defined by

$$
F_{k}=\frac{\sum_{x=0}^{M} A_{k}(x) I(x)}{\sum_{x=0}^{M} A_{k}(x)}, k=0, \ldots, m .
$$

We can approximately reconstruct function $I$ using inverse formula

$$
O(x)=\sum_{k=0}^{m} F_{k} A_{k}(x), x=0, \ldots, M,
$$

where $O$ is the reconstructed function.

Let us introduce F-transform of a 2D image $I$ that is considered as a function $I:[0, M] \times[0, N] \rightarrow$ $[0,255]$ where $[0, M]=\{0,1,2, \ldots, M\} ;[0, N]=$ $\{0,1,2, \ldots, N\}$. It is assumed that the image is grayscale and defined at points (pixels) that belong to the set $P$, where $P=\{(x, y) \mid x=$ $0,1, \ldots, M ; y=0,1, \ldots, N\}$.

Let $A_{0}, \ldots, A_{m}$ and $B_{0}, \ldots, B_{n}$ be basic functions, $A_{0}, \ldots, A_{m}:[0, M] \rightarrow[0,1]$ be fuzzy partition of $[0, M]$ and $B_{0}, \ldots, B_{n}:[0, N] \rightarrow[0,1]$ be fuzzy partition of $[0, N]$. Assume that the set of pixels $P$ is sufficiently dense with respect to the chosen partitions. This means that for all $k \in 0, \ldots, m(\exists x \in[0, M]) A_{k}(x)>0$, and for all $l \in 0, \ldots, n(\exists y \in[0, N]) B_{l}(y)>0$.

We say that the $m \times n$-matrix of real numbers $\left[F_{k l}\right]$ is called the (discrete) F-transform of $I$ with respect to $\left\{A_{0}, \ldots, A_{m}\right\}$ and $\left\{B_{0}, \ldots, B_{n}\right\}$ if for all $k=0, \ldots, m, l=0, \ldots, n$,

$$
F_{k l}=\frac{\sum_{y=0}^{N} \sum_{x=0}^{M} I(x, y) A_{k}(x) B_{l}(y)}{\sum_{y=0}^{N} \sum_{x=0}^{M} A_{k}(x) B_{l}(y)} .
$$

The elements $F_{k l}$ are called components of the $F$ transform. The inverse F-transform identified with the output image $O: P \rightarrow[0,255]$ of the function $I$ with respect to $\left\{A_{0}, \ldots, A_{m}\right\}$ and $\left\{B_{0}, \ldots, B_{n}\right\}$ is defined as follows

$$
O(x, y)=\sum_{k=0}^{m} \sum_{l=0}^{n} F_{k l} A_{k}(x) B_{l}(y) .
$$

The function $O$ can approximate the original function $I$ on the whole domain $P$ with a given precision.

For the application described in this paper, we using the same radius $h$ for basic functions $A$ and $B$. Fig. 3 represents an example of the coverage.

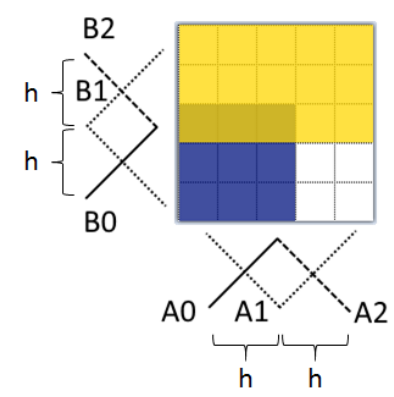

Figure 3: Solid basic function stands for $A_{0}, B_{0}$, dotted for $A_{1}, B_{1}$ and dashed for $A_{2}, B_{2}$.

\subsection{F-transform computation based on a partition matrix}

Let us demonstrate the matrix representation of (1) described in [9]. Direct F-transform is computed as follows 


$$
\mathbf{F}_{m}[I]=\left(F_{0}, \ldots, F_{m}\right)=\left(\frac{\left(I A^{\prime}\right)_{0}}{a_{0}}, \ldots, \frac{\left(I A^{\prime}\right)_{m}}{a_{m}}\right),
$$

where $a_{k}=\sum_{x=0}^{M} A_{k}(x), k=0, \ldots, m$. The partition matrix $A^{\prime}$ is composed based on the vectors $A_{k}$ as follows

$$
\begin{aligned}
& A^{\prime}=\left(\begin{array}{cccc}
A_{0}^{0} & 0 & 0 & 0 \\
\vdots & 0 & 0 & \vdots \\
A_{0}^{h-2} & 0 & 0 & 0 \\
A_{0}^{h-1} & 0 & 0 & 0 \\
A_{0}^{h} & A_{1}^{0} & \ldots & 0 \\
A_{0}^{h+1} & \vdots & \vdots & 0 \\
A_{0}^{h+2} & A_{1}^{h-2} & \ldots & 0 \\
\vdots & A_{1}^{h-1} & \ldots & 0 \\
A_{0}^{2 h+1} & A_{1}^{h} & \ldots & A_{m}^{0} \\
0 & A_{1}^{h+1} & \ldots & \vdots \\
0 & A_{1}^{h+2} & \ldots & A_{m}^{h-2} \\
0 & \vdots & \ldots & A_{m}^{h-1} \\
0 & A_{1}^{2 h+1} & \ldots & A_{m}^{h} \\
0 & 0 & \ldots & A_{m}^{h+1} \\
0 & 0 & \ldots & A_{m}^{h+2} \\
0 & 0 & \ldots & \vdots \\
0 & 0 & \ldots & A_{m}^{2 h+1}
\end{array}\right) \\
& I=\left(\begin{array}{lllll}
I_{0} & I_{1} & I_{2} & \ldots & I_{M}
\end{array}\right),
\end{aligned}
$$

where $A_{k}^{h-j}=A_{k}^{h+j}$ and $I_{x}=I(x)$.

In case of a $h$-uniform fuzzy partition, only one function $A$ is used and translated for the whole range. This function is called generating function. It leads to condition $A_{k}(k h \pm j)=A( \pm j)$ where $h$ is the radius of a basic function. Let us denote that matrix $A^{\prime}$ have to be adjusted based on the input image $I$ and a basic function $A$. Concrete example for basic function $A=(0, .5,1, .5,0), m=3$ and image $I=(0,10,15,18,23,20,19,8)$ leads to

$$
\begin{gathered}
A^{\prime}=\left(\begin{array}{ccccc}
1 & 0 & 0 & 0 & 0 \\
.5 & .5 & 0 & 0 & 0 \\
0 & 1 & 0 & 0 & 0 \\
0 & .5 & .5 & 0 & 0 \\
0 & 0 & 1 & 0 & 0 \\
0 & 0 & .5 & .5 & 0 \\
0 & 0 & 0 & 1 & 0 \\
0 & 0 & 0 & .5 & .5
\end{array}\right) \\
I A^{\prime}=(0,10,15,18,23,20,19,8) A^{\prime},
\end{gathered}
$$

which is equal to $I A^{\prime}=(5,29,42,33,4)$. Direct Ftransform is equal to

$$
\mathbf{F}_{m}[I]=(3.33,14.5,21,16.5,8) .
$$

The image $O$ approximated by the inverse $\mathrm{F}$ transform is equal to matrix and vector multiplication as follows

$$
O=A^{\prime} \mathbf{F}_{m}^{T}
$$

which leads to

$$
O=(3.33,8.92,14.5,17.75,21,18.75,16.5,12.25)^{T} .
$$

\subsection{F-transform as a convolution}

Let us assume that the interval $[0, M]$ is $h$-uniformly partitioned by fuzzy sets $A_{0}, \ldots, A_{m}, I$ is a $1 \mathrm{D}$ discrete function, and the F-transform of the discrete function $I$ is given by $\mathbf{F}_{m}[I]$ with components obtained by (1).

It is easy to see that if the fuzzy partition $A_{0}, \ldots, A_{m}$ of $[0, M]$ is $h$-uniform, then there exists an even function

$$
A:[-h, h] \rightarrow[0,1],
$$

such that for all $k=0, \ldots, m$,

$$
A_{k}(x)=A\left(x-x_{k}\right)=A\left(x_{k}-x\right), x \in\left[x_{k-1}, x_{k+1}\right] .
$$

As was mentioned earlier, we call $A$ a generating function of an $h$-uniform fuzzy partition. The example of a triangular generating function $A$ and the respective $h$-uniform fuzzy partition is given in Fig. 4.

Generating function A and Fuzzy Partition

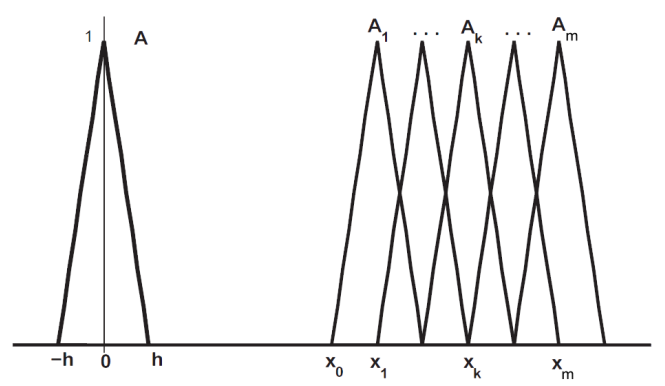

Figure 4: Generating function $A$ of an $h$-uniform fuzzy partition.

For image processing, where are pixels equidistant (see [7]), there exists a constant $c>0$ such that for all $k=0, \ldots, m$,

$$
\sum_{x=0}^{M} A_{k}(x)=c .
$$

By (5) and (4), the expression (1) can be rewritten as follows

$$
F_{k}=\frac{\sum_{x=0}^{M} A\left(x_{k}-x\right) I(x)}{c} ; k=0, \ldots, m .
$$

Let us consider $F_{k}$ as a value of a discrete function $F$, defined on the set $\mathbf{Z}_{m}=\{0, \ldots, m\}$ with values 
from $\mathbb{R}$ such that $F: \mathbf{Z}_{m} \rightarrow \mathbb{R}$ and $F(k)=F_{k}$. We will use (6) for an analytic extension of $F$ from $\mathbf{Z}_{m}$ to $\mathbf{Z}_{l}=\{0,1, \ldots, M\}$, so that

$$
F(t)=\frac{\sum_{x=0}^{M} A(t-x) I(x)}{c} ; t=0, \ldots, M .
$$

Finally, we will normalize values of $A$ dividing them by $c$ and keep the same denotation $A$ for the normalized function. Then without loss of generality, we will continue working with the below given expression for $F$

$$
F(t)=\sum_{x=0}^{M} A(t-x) I(x) ; t=0, \ldots, M .
$$

Analyzing (8), we can see that the function $F$ : $\mathbf{Z}_{l} \rightarrow \mathbb{R}$ is a convolution (see e.g., [10], [7]) of two discrete functions $A$ and $I$. Let us recall that $F$ contains the $F$-transform components $F_{k}, k=0, \ldots, m$ among its values.

Based on the relationship between the convolution and the F-transform, we can say that features of the convolution such as

$$
\begin{aligned}
I * g & =g * I, \\
I *(f * g) & =(I * f) * g, \\
\left(I_{1}+I_{2}\right) * g & =\left(I_{1} * g\right)+\left(I_{2} * g\right),
\end{aligned}
$$

applies also for F-transform.

\section{Convolution}

The operation of convolution is defined as

$$
(I * g)(x, y)=\sum_{i, j=-k}^{k} I(x-i, y-j) \cdot g(i, j),
$$

where $I$ is an image and $g$ is a kernel function. In image processing the size of the kernel is typically $3 \times 3$. Some examples of the kernels are in Fig. 5 .

\begin{tabular}{|l|l|l|}
\hline 1 & 1 & 1 \\
\hline 1 & 1 & 1 \\
\hline 1 & 1 & 1 \\
\hline
\end{tabular}

(a) Mean

\begin{tabular}{|l|l|l|}
\hline 1 & 2 & 1 \\
\hline 2 & 4 & 2 \\
\hline 1 & 2 & 1 \\
\hline
\end{tabular}

(b) Gaussian

\begin{tabular}{|l|l|l|}
\hline-1 & 0 & 1 \\
\hline-2 & 0 & 2 \\
\hline-1 & 0 & 1 \\
\hline
\end{tabular}

(c) Sobel
Figure 5: Various kernels use for convolution.

A graphical representation of the computation based on (9) is in Fig. 6. The figure includes kernel $g$, input image $I$ and output image $O$.

For the marked area in Fig. 6 is computation as follows

$$
\begin{aligned}
O(1,2) & =\frac{1}{9}(56 \cdot 1+80 \cdot 1+67 \cdot 1+57 \cdot 1+ \\
& 77 \cdot 1+76 \cdot 1+59 \cdot 1+77 \cdot 1+74 \cdot 1)= \\
& 69
\end{aligned}
$$

\begin{tabular}{|l|l|l|}
\hline 1 & 1 & 1 \\
\hline 1 & 1 & 1 \\
\hline 1 & 1 & 1 \\
\hline
\end{tabular}

(a) Kernel

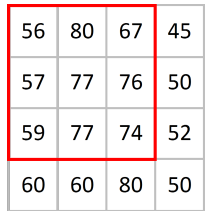

(b) Input

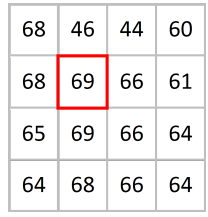

(c) Output
Figure 6: Illustration of the convolution operation.

Next step is to shift the kernel by one position to the right, repeat a computation, etc. On the edge, where are less than 9 known pixels, only the known ones are applied.

\subsection{Convolution computation based on the matrix}

It is possible to perform the computation of the convolution operation by Toeplitz matrix and vector multiplication

$$
O=g * I=g^{\prime} I
$$

where

$$
\begin{aligned}
g^{\prime} & =\left(\begin{array}{ccccc}
g_{0} & 0 & \ldots & 0 & 0 \\
g_{1} & g_{0} & \ldots & \vdots & \vdots \\
g_{2} & g_{1} & \ldots & 0 & 0 \\
\vdots & g_{2} & \ldots & g_{0} & 0 \\
g_{m-1} & \vdots & \ldots & g_{1} & g_{0} \\
g_{m} & g_{m-1} & \vdots & \vdots & g_{1} \\
0 & g_{m} & \ldots & g_{m-2} & \vdots \\
0 & 0 & \cdots & g_{m-1} & g_{m-2} \\
\vdots & \vdots & \vdots & g_{m} & g_{m-1} \\
0 & 0 & 0 & \cdots & g_{m}
\end{array}\right) \\
I & =\left(\begin{array}{c}
I_{0} \\
I_{1} \\
I_{2} \\
\vdots \\
I_{M}
\end{array}\right) .
\end{aligned}
$$

For instance, the kernel $g=(1,-2,1)$ and the image $I=(0,10,15,18,23,20,19,8)^{T}$ leads to

$$
\begin{aligned}
& g^{\prime}=\left(\begin{array}{cccccccc}
1 & 0 & 0 & 0 & 0 & 0 & 0 & 0 \\
-2 & 1 & 0 & 0 & 0 & 0 & 0 & 0 \\
1 & -2 & 1 & 0 & 0 & 0 & 0 & 0 \\
0 & 1 & -2 & 1 & 0 & 0 & 0 & 0 \\
0 & 0 & 1 & -2 & 1 & 0 & 0 & 0 \\
0 & 0 & 0 & 1 & -2 & 1 & 0 & 0 \\
0 & 0 & 0 & 0 & 1 & -2 & 1 & 0 \\
0 & 0 & 0 & 0 & 0 & 1 & -2 & 1 \\
0 & 0 & 0 & 0 & 0 & 0 & 1 & -2 \\
0 & 0 & 0 & 0 & 0 & 0 & 0 & 1
\end{array}\right) \\
& O=g^{\prime}(0,10,15,18,23,20,19,8)^{T},
\end{aligned}
$$


which is equal to

$$
O=(0,10,-5,-2,2,-8,2,-10,3,8)^{T} .
$$

\section{Comparison}

Both methods (convolution and F-transform) are possible to be computed using matrix and vector multiplication. There is a similarity in the pattern for matrix $g^{\prime}$ and $A^{\prime}$ shown in Fig. 7 and Fig. 8 . For simplicity, let us ignore special conditions at the image borders.

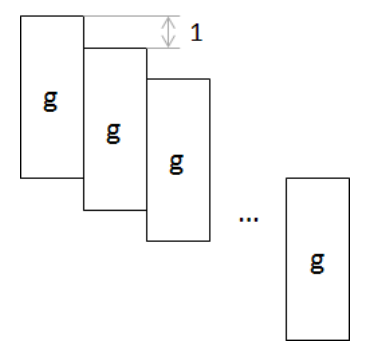

Figure 7: Pattern for the convolution matrix $g^{\prime}$ using kernel $g$.

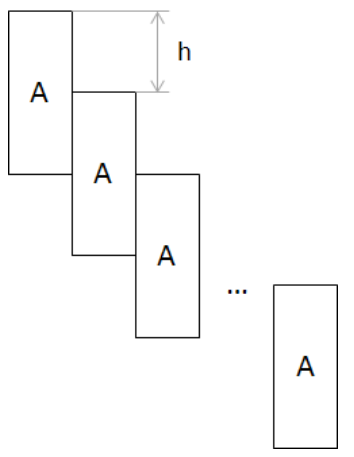

Figure 8: Pattern for the F-transform matrix $A^{\prime}$ using basic function $A$.

We can see that the columns are shifted copies of the vectors $g$ and $A$. The difference is in a shift distance between two neighbour copies. The distance is 1 for convolution and radius of the basic function $h$ for F-transform. Let us show some examples of the convolution and the F-transform applied to a $2 \mathrm{D}$ image. Tab. 1 shows input image with the mean kernel.

\begin{tabular}{|l|l|l|}
\hline 1 & 1 & 1 \\
\hline 1 & 1 & 1 \\
\hline 1 & 1 & 1 \\
\hline
\end{tabular}

\begin{tabular}{|l|l|l|}
\hline 5 & 6 & 1 \\
\hline 8 & 2 & 3 \\
\hline 1 & 4 & 2 \\
\hline
\end{tabular}

Table 1: The mean kernel and the input image.

Value of the middle pixel is up to two decimal places

$$
O(1,1)=3.56 \text {. }
$$

For F-transform and the generating function

$$
A=(1,1,1)
$$

the input configuration is as follows

\begin{tabular}{c||c|c|c|}
\hline 1 & 5 & 6 & 1 \\
\hline 1 & 8 & 2 & 3 \\
\hline 1 & 1 & 4 & 2 \\
\hline \hline & 1 & 1 & 1
\end{tabular}

The direct F-transform component corresponding to the central pixel is equal to

$$
F_{00}=\frac{32}{9}=3.56 \text {. }
$$

We can see that the results are equal. Kernel $g$ correlates with the generating function $A$ as follows

$$
g=A^{T} A
$$

Tab. 2 shows input image and the Gaussian kernel.

\begin{tabular}{|l|l|l|}
\hline 1 & 2 & 1 \\
\hline 2 & 4 & 2 \\
\hline 1 & 2 & 1 \\
\hline
\end{tabular}

\begin{tabular}{|l|l|l|}
\hline 5 & 6 & 1 \\
\hline 8 & 2 & 3 \\
\hline 1 & 4 & 2 \\
\hline
\end{tabular}

Table 2: The Gaussian kernel and the input image.

The convolution value of the central pixel is

$$
O(1,1)=3.69 \text {. }
$$

We will decompose and normalize the Gaussian kernel and extract the generating function $A=$ $(.5,1, .5)$. Then the F-transform component at the central node corresponds to the scheme

\begin{tabular}{c||c|c|c|}
\hline .5 & 5 & 6 & 1 \\
\hline 1 & 8 & 2 & 3 \\
\hline .5 & 1 & 4 & 2 \\
\cline { 2 - 4 } & .5 & 1 & .5
\end{tabular}

and is equal to

$$
F_{00}=\frac{32}{9}=3.69 .
$$

A comparison between a basic convolution blurring and a basic F-transform blurring is illustrated in Fig. 1 and Fig. 2 where is denoted as an unsophisticated noise reduction. There are used the Gaussian kernel with $k=2$ and the linear generating function with $h=2$. For the generating function, we can write without 0 borders

$$
\left(\begin{array}{ccc}
.25 & .5 & .25 \\
.5 & 1 & .5 \\
.25 & .5 & .25
\end{array}\right)=\left(\begin{array}{c}
.5 \\
1 \\
.5
\end{array}\right)(.5,1, .5)
$$

with respect to (10).

We can say that output images look very similar based on the Fig. 1 visual comparison. For the 
numeric comparison we choose the quality measure RMSE. This measure estimates closeness between an original image with the processed one, where 0 value denotes equality. The RMSE value for convolution with the Gaussian kernel is 7.27 and for F-transform is 6.62. These two RMSE values show that the convolution and the F-transform produces similar outputs. Moreover, the F-transform is closer to the original one.

\section{Conclusion}

We have shown that the direct F-transform of a discrete function can be equal to a convolution applied to this function. The kernel correlates with a generating function of the corresponding fuzzy partition. For a discrete function of two arguments (image), we compared the convolution with the Gaussian kernel and the related to it direct and inverse F-transform. We showed that both transformations remove noise and estimated their RMSE quality. We gave various numerical examples that confirm our conclusions that there is a connection between convolution operation and F-transform. The behavior comparable with a convolution let us use its advantages and features which is currently under deeper investigation.

\section{References}

[1] Saeed V Vaseghi. Advanced digital signal processing and noise reduction. John Wiley \& Sons, 2008.

[2] Zhao Yu-qian, Gui Wei-hua, Chen Zhen-cheng, Tang Jing-tian, and Li Ling-yun. Medical images edge detection based on mathematical morphology. In Engineering in Medicine and Biology Society, 2005. IEEE-EMBS 2005. 27th Annual International Conference of the, pages 6492-6495. IEEE, 2006.

[3] MI Rajab, MS Woolfson, and SP Morgan. Application of region-based segmentation and neural network edge detection to skin lesions. Computerized Medical Imaging and Graphics, 28(1):61-68, 2004.

[4] Irina Perfilieva, Petra Hod'áková, and Petr Hurtík. $\quad F^{1}$-transform edge detector inspired by canny's algorithm. In Advances on Computational Intelligence, pages 230-239. Springer, 2012.

[5] Pavel Vlašánek and Irina Perfilieva. Influence of various types of basic functions on image reconstruction using F-transform. In European Society for Fuzzy Logic and Technology, pages 497 - 502. Atlantis Press, 2013.

[6] Marek Vajgl, Irina Perfilieva, and Petra Hod'áková. Advanced f-transform-based image fusion. Advances in Fuzzy Systems, 2012:4, 2012 .
[7] Irina Perfilieva and Petra Hodáková. Fuzzy and Fourier transforms. In EUSFLAT Conf., pages 452-456, 2011.

[8] Irina Perfilieva. Fuzzy transforms: Theory and applications. Fuzzy sets and systems, 157(8):993-1023, 2006.

[9] Irina Perfilieva, Nadezhda Yarushkina, Tatiana Afanasieva, and Anton Romanov. Time series analysis using soft computing methods. International Journal of General Systems, 42(6):687-705, 2013.

[10] George Bachmann, Lawrence Narici, and Edward Beckenstein. Fourier and wavelet analysis. Springer, 2000. 